Cahiers $d u$ MONDE RUSSE

\section{Cahiers du monde russe}

Russie - Empire russe - Union soviétique et États indépendants

50/2-3 | 2009

L'Europe orientale, 1650-1730. Crises, conflits et renouveau

\title{
Authoritarian BacklashThomas AMBROSIO
}

, Surrey : Ashgate, 2009, 240 p.

\section{Ioulia Shukan}

\section{(2) OpenEdition}

\section{Journals}

Édition électronique

URL : https://journals.openedition.org/monderusse/9778

DOI : $10.4000 /$ monderusse.9778

ISSN : $1777-5388$

Éditeur

Éditions de l'EHESS

Édition imprimée

Date de publication : 15 septembre 2009

ISBN : 978-2-7132-2260-3

ISSN : $1252-6576$

Référence électronique

Ioulia Shukan, "Authoritarian BacklashThomas AMBROSIO », Cahiers du monde russe [En ligne], 50/2-3 | 2009, mis en ligne le 14 janvier 2013, consulté le 03 septembre 2022. URL : http:// journals.openedition.org/monderusse/9778; DOI : https://doi.org/10.4000/monderusse. 9778

Ce document a été généré automatiquement le 3 septembre 2022

Tous droits réservés 


\section{Authoritarian BacklashThomas AMBROSIO}

, Surrey : Ashgate, 2009, 240 p.

Ioulia Shukan

\section{Thomas AMBROSIO, Authoritarian Backlash, Russian Resistance to Democratization in the Former Soviet Union, Surrey : Ashgate, 2009, $240 \mathrm{p}$.}

1 Si la quatrième vague de démocratisation qui avait secoué l'espace postsoviétique dans les années 2000 à la faveur des révolutions de couleur intervenues en Géorgie en 2003, en Ukraine en 2004 et au Kirghizstan en 2005 a suscité des espoirs d'installation durable de la démocratie, elle a dans le même temps concrétisé la menace d'une contagion démocratique dans la zone, provoquant en retour des réactions brutales de la part des régimes autoritaires. C'est l'analyse de ces réactions à partir de l'exemple de la Russie de Vladimir Putin qui se trouve au cœur de l'ouvrage de Thomas Ambrosio, professeur de sciences politiques à l'université d'État du North Dakota.

2 Tout en s'inscrivant dans la continuité du paradigme théorique de la transition démocratique, et notamment des analyses de l'exportation de la démocratie à l'échelle mondiale, ce livre a pour ambition d'y apporter une nouvelle contribution. Il accorde ainsi une attention particulière non pas aux succès de la démocratisation, mais plutôt à ses échecs dans l'espace postsoviétique, défendant l'idée que ses vagues successives peuvent se briser et refluer (ch. I). De même, s'il admet l'importance des acteurs transnationaux et donc des facteurs internationaux dans les processus de promotion extérieure de la démocratie, il défend cependant l'idée que ces processus sont interactifs et conduisent les États autoritaires à contre-attaquer face aux pressions internationales dont ils font l'objet.

Le chapitre II identifie ainsi cinq stratégies principales de résistance à la promotion de la démocratie développées par les autorités russes : isoler, redéfinir, promouvoir, subvertir et coopérer avec les États qui partagent leurs intérêts, stratégies ayant pour objet à la 
fois de prévenir la reproduction du modèle de révolution de couleur dans d'autres États postsoviétiques - et donc la contagion démocratique dans l'ex-URSS -, et de créer un environnement régional favorable à l'affirmation de l'autoritarisme. Ces cinq stratégies sont analysées dans les chapitres IV à VIII. Leur examen détaillé est précédé d'un bref rappel (ch. III) des évolutions politiques que la Russie a connues dans les années 1990-2000 et qui vont d'une courte période démocratique sous la présidence de Boris El 'cin au basculement dans l'autoritarisme centralisateur à la suite de l'élection à la présidence de Vladimir Putin.

4 La première tactique (ch. IV) vise à isoler les forces transnationales impliquées dans l'exportation de la démocratie et à empêcher leur action en Russie. Elle comprend trois séries de mesures. Il s'agit d'abord du placement des ONG internationales de défense des droits de l'homme sous un contrôle étatique strict par la loi de janvier 2006. Il est ensuite question du travail de discréditation des missions d'observation électorales conduites par les instances européennes (Bureau des institutions démocratiques et des droits de l'homme de l'OSCE, Conseil de l'Europe), qui avaient pointé dans les années 2000 le caractère non équitable des élections en Russie. Dans le cas présent, les techniques employées vont de la dénonciation de la partialité des conclusions produites par les observateurs européens à la mise en place de missions d'observation électorale alternatives dans le cadre de la Communauté des États indépendants (CEI). Il s'agit, enfin, de la création de mouvements de jeunes, loyaux aux autorités, chargés du travail de mobilisation de la jeunesse en faveur du régime de Putin et de subversion des activités conduites par de jeunes opposants russes.

5 À partir de l'analyse du discours officiel, le chapitre $\mathrm{V}$ retrace la stratégie de redéfinition du concept même de démocratie, dont la valeur universelle est remise en cause par une argumentation de type culturaliste, mettant l'accent sur la diversité des voies nationales de démocratisation. Ici, le concept de "démocratie souveraine» forgé au sein de l'administration présidentielle est appelé à légitimer une démocratie " administrée » par le haut et faisant primer la raison d'État sur les libertés publiques, conformément à la tradition historique russe et, partant, à récuser les critiques occidentales sur l'état de la démocratie en Russie. Parmi les autres manœuvres rhétoriques recensées, on trouve la stigmatisation de la promotion internationale de la démocratie en tant que politique néocoloniale de l'Occident, ou encore la dénonciation du laxisme des instances internationales ou européennes face aux problèmes concernant les droits de l'homme dans les pays occidentaux, alors que ces instances dénoncent ces mêmes problèmes ailleurs (tactique partiale du « deux poids, deux mesures»).

6 Les chapitres VI et VII présentent deux politiques menées par les autorités russes au niveau régional à l'égard des pays membres de la CEI. Il s'agit d'abord de la stratégie de soutien aux régimes autoritaires de la zone, analysée à partir des relations privilégiées entretenues avec la Biélorussie d'Aleksandr Lukašenko. D'après Ambrosio, la Russie aurait fourni au régime de Lukašenko les ressources diplomatiques, politiques, économiques et militaires nécessaires pour résister à l'isolement international et aux pressions dont ce régime faisait l'objet dans les années 2000, garantissant ainsi sa survie sur le long terme. Une autre manœuvre consiste à ébranler par divers moyens (pressions économiques, attaques rhétoriques, soutien à certains groupes ethniques) les régimes démocratiques installés en Géorgie et en Ukraine à la suite des révolutions de couleur, un échec des processus de démocratisation dans ces pays étant susceptible de remettre en cause les aspirations régionales à la démocratie, mais aussi de compromettre les chances 
d'intégration de ces pays à l'OTAN. À cet égard, l'ouvrage établit un lien de causalité direct entre les objectifs de politique extérieure des États postsoviétiques et la nature de leur régime politique, de même qu'entre la politique extérieure de la Russie et le type de régime de ses voisins.

7 La dernière stratégie de résistance à la diffusion démocratique (ch. VIII) consiste dans la multiplication des coopérations avec d'autres pays autoritaires de la région, notamment dans le cadre de l'Organisation de coopération de Shanghai. Réunissant en son sein Russie, Chine, Kazakhstan, Kirghizstan, Ouzbékistan et Tadjikistan, cette organisation a pour objectif de garantir la stabilité dans la région à travers la lutte contre le terrorisme, le séparatisme et surtout l'extrémisme, entendu comme toute tentative de renversement par la force de l'ordre politique établi. Elle défend également une vision relativiste des droits de l'homme et de la démocratie, le contenu de ces deux concepts devant être adapté aux caractéristiques nationales de chaque pays.

8 Enfin le chapitre IX revient sur le cycle électoral de 2007-2008 en Russie pour constater l'efficacité des stratégies de neutralisation des acteurs de l'exportation démocratique: exposition des antennes locales d'ONG internationales à l'arbitraire des administrations chargées de vérifier leurs activités statutaires et fiscales ; obstacles posés au déploiement des observateurs de l'OSCE aussi bien pour les élections législatives de décembre 2007 que présidentielles de mars 2008; encadrement du comportement politique de la jeunesse par des mouvements de jeunes loyaux au régime. Au regard de ces succès constatés tant au plan national que régional, l'auteur conclut au reflux de la vague de démocratisation en Russie et, de manière plus générale, dans l'espace postsoviétique ainsi qu'au déferlement d'une nouvelle vague d'autoritarisme sur cette zone.

Si l'ouvrage analyse de manière détaillée les réactions du gouvernement russe face à la menace de diffusion démocratique dans la région et contribue à une meilleure compréhension de la persistance d'un régime autoritaire dans l'ex-URSS, il suscite cependant quelques questionnements. D'abord, cette résistance à la promotion de la démocratie est principalement abordée sous l'angle de la politique extérieure de la Russie. À cet égard, on peut déplorer que l'ouvrage prête si peu d'attention notamment aux stratégies coercitives déployées par l'État russe à l'encontre des acteurs locaux de l'opposition à l'intérieur même du pays, stratégies qui semblent jouer un rôle tout aussi important dans la prévention des révolutions de couleur.

10 Ensuite, on peut s'interroger sur le lien de causalité directe, établi de manière quelque peu rapide par l'auteur, entre les priorités de politique extérieure des États postsoviétiques et la nature de leur régime politique. Si le respect des principes démocratiques est l'une des conditions préalables à l'adhésion à l'OTAN et à l'Union européenne, le manquement à ces principes n'implique pas automatiquement une politique extérieure ouvertement prorusse. De ce point de vue, la Géorgie fournit un bon exemple : une sensible remise en cause des principes de la compétition politique (respect de l'opposition, élection libre et équitable) sous la présidence de Saakašvili ne s'y est pas soldée par le réalignement de ce pays sur la Russie. De la même manière, de nouvelles coopérations récemment engagées entre l'Union européenne et la Biélorussie n'ont pas conduit à une quelconque libéralisation politique du régime de Lukašenko.

11 Notre dernière interrogation porte, enfin, sur les motivations réelles des cinq stratégies de résistance de la Russie à la quatrième vague de démocratisation. Doivent-elles être attribuées, comme l'auteur le prétend, à la menace de démocratisation des pays limitrophes et donc de contagion démocratique dans l'espace postsoviétique, 
susceptible, à terme, de remettre en cause les positions du pouvoir russe ; ou plutôt au risque de voir ces pays échapper à ce que la Russie considère comme sa sphère d'influence? À cet égard, Thomas Ambrosio reconnaît lui-même les limites de son analyse en admettant l'impossibilité de séparer nettement les considérations politiques liées aux impératifs de sécurité régionale des préoccupations d'ordre géopolitique. 